\title{
Expertise in action observation: recent neuroimaging findings and future perspectives
}

\author{
Luca Turella ${ }^{1 *}$, Moritz F. Wurm ${ }^{1}$, Raffaele Tucciarelli ${ }^{1}$ and Angelika Lingnau ${ }^{1,2}$ \\ ${ }^{1}$ Center for Mind/Brain Sciences (C/MeC), University of Trento, Trento, Italy \\ 2 Department of Cognitive Sciences, University of Trento, Trento, Italy \\ *Correspondence: luca.turella@gmail.com; luca.turella@unitn.it \\ Edited by: \\ Robert Langner, Heinrich Heine University Düsseldorf, Germany \\ Reviewed by: \\ Svenja Caspers, Research Centre Juelich, Germany
}

Keywords: fMRI, expertise, action observation network, mentalizing system

\section{INTRODUCTION}

In everyday life, we continuously interact with other individuals. Understanding actions of other people, i.e., the ability to distinguish between different actions, such as passing over vs. threatening someone with a knife, has been crucial for the survival of our species and is a fundamental capability for our social interactions.

Neuroimaging studies investigated the neural substrates subtending action perception using a variety of techniques, ranging from univariate analysis of fMRI data (Brass et al., 2007; Gazzola et al., 2007; De Lange et al., 2008; Gazzola and Keysers, 2009; Turella et al., 2009a, 2012; Wurm et al., 2011; Wurm and Schubotz, 2012; Wurm et al., 2012; Lingnau and Petris, 2013), to fMRI repetition suppression (Dinstein et al., 2007; Chong et al., 2008; Lingnau et al., 2009; Kilner et al., 2009) and multivoxel pattern analysis (MVPA; Dinstein et al., 2008a; Oosterhof et al., 2010, 2012). These studies reported the consistent recruitment of a number of regions, generally assumed as pertaining to two different networks, typically referred to as the action observation network $(\mathrm{AON})$ and the mentalizing system (Figure 1A). Both networks have been advocated to be involved in action understanding (Brass et al., 2007; De Lange et al., 2008; Van Overwalle, 2009; Van Overwalle and Baetens, 2009; Wurm et al., 2011), but their precise roles and their causal involvement are strongly debated (Dinstein et al., 2008b; Mahon and Caramazza, 2008; Hickok, 2009; Turella et al., 2009b; Rizzolatti and Sinigaglia, 2010).

In homology with monkey neurophysiological studies, three regions have been proposed to form the human AON (Rizzolatti and Craighero, 2004; Rizzolatti and Sinigaglia, 2010; see Figure 1A). This "core" AON was defined as comprising (i) the ventral premotor cortex (PMV) together with the posterior part of the inferior frontal gyrus (pIFG), (ii) the anterior inferior parietal lobule (aIPL) and (iii) the superior temporal sulcus (STS). Human neuroimaging studies suggested the recruitment of several additional areas that were incorporated in an "extended" version of the AON (Gazzola and Keysers, 2009; Caspers et al., 2010; see Figure 1A).

The mentalizing system has been identified in human neuroimaging studies investigating social cognition tasks, such as intention and beliefs attribution about the self or others, while observing actionrelated stimuli (Van Overwalle, 2009). The regions consistently assigned to this network are the medial prefrontal cortex (mPFC) and the temporo-parietal junction (TPJ) (Figure 1A), and less often also the precuneus and the posterior cingulate cortex (Amodio and Frith, 2006; Brass et al., 2007; De Lange et al., 2008; Van Overwalle, 2009; Van Overwalle and Baetens, 2009).

The first description of the involvement of sensorimotor regions during action perception started with the discovery of mirror neurons in the ventral premotor cortex in macaque monkeys (Di Pellegrino et al., 1992). These visuomotor neurons responded both while the monkey executed or observed similar actions and were later described also within the monkey inferior parietal lobule (Fogassi et al., 2005). Note that both regions also contain neurons with motor-only and visual-only properties (Gallese et al., 1996, 2002).
Following their discovery, motor theories of action understanding proposed that mirror neurons might provide the basis for a matching mechanism between what we observe and what we can perform allowing the understanding of observed actions in motoric terms (Rizzolatti et al., 2001). Even if this hypothesis is strongly debated (Jacob and Jeannerod, 2005; Mahon and Caramazza, 2008; Hickok, 2009, 2013), a similar homologue mechanism has been proposed to exist in the human AON (Rizzolatti et al., 2001; Rizzolatti and Craighero, 2004; Rizzolatti and Sinigaglia, 2010).

In this brief overview, we will first describe previous fMRI studies that investigated how motor experience affects activation within the $\mathrm{AON}$, and to which degree these studies allow drawing conclusions about the role of this network in action understanding. As the majority of the studies investigated only the $\mathrm{AON}$ and given the limited scope of this Opinion, we will focus on this network, even if our considerations might also hold true for other areas. We will then try to delineate how future studies might exploit motor expertise as a tool for gaining insights into the neural basis of action understanding.

\section{RECENT NEUROIMAGING FINDINGS ON MOTOR EXPERTISE IN ACTION OBSERVATION}

Following motor theories of action understanding, changes in motor repertoire should modify the brain response within the AON while observing these newly acquired actions. Starting from this assumption, most studies on expertise investigated how the acquisition of a skilled action, such as sport or 
A

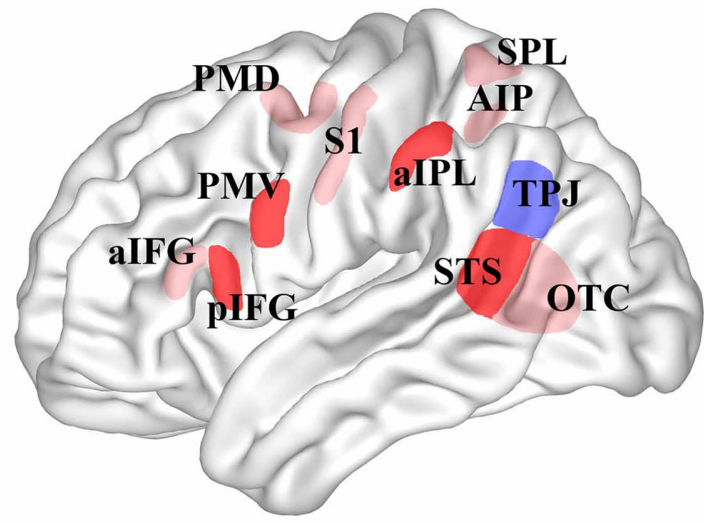

Core AON

B
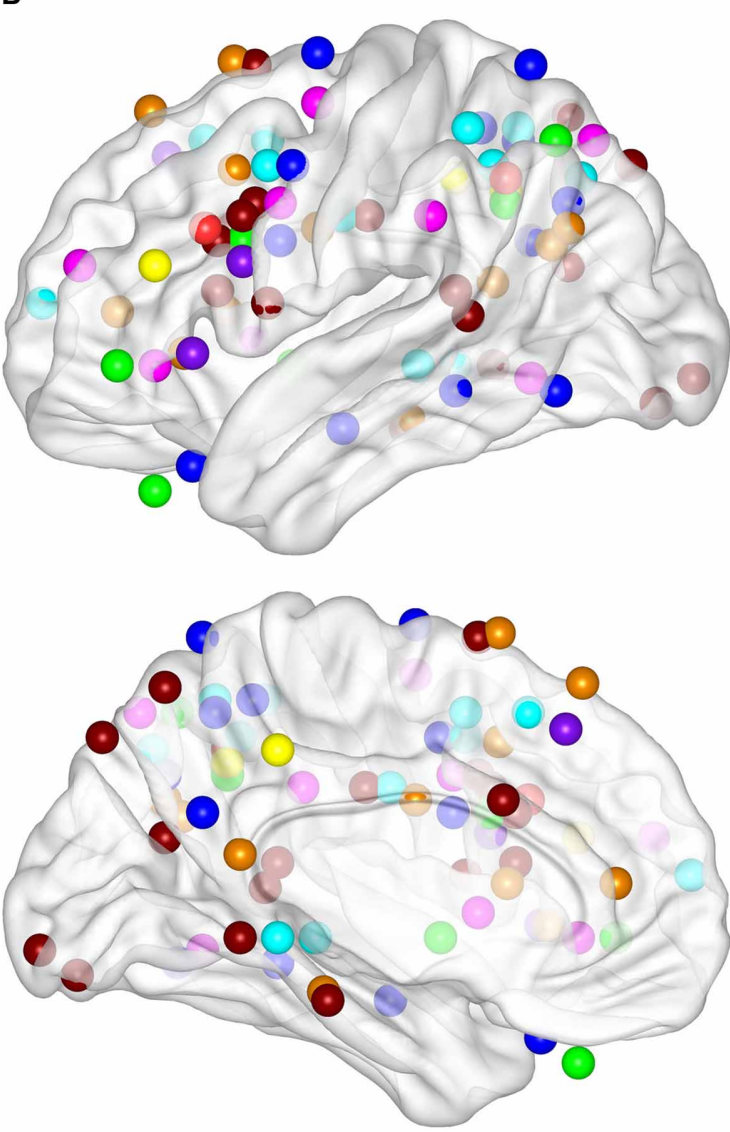

Archery expertise - Kim et al., 2011

Badminton expertise - Wright et al., 2010

Badminton expertise - Wright et al., 2011

Basketball expertise - Abreu et al., 2012

Dance expertise - Calvo-Merino et al., 2005

Dance expertise - Calvo-Merino et al., 2006

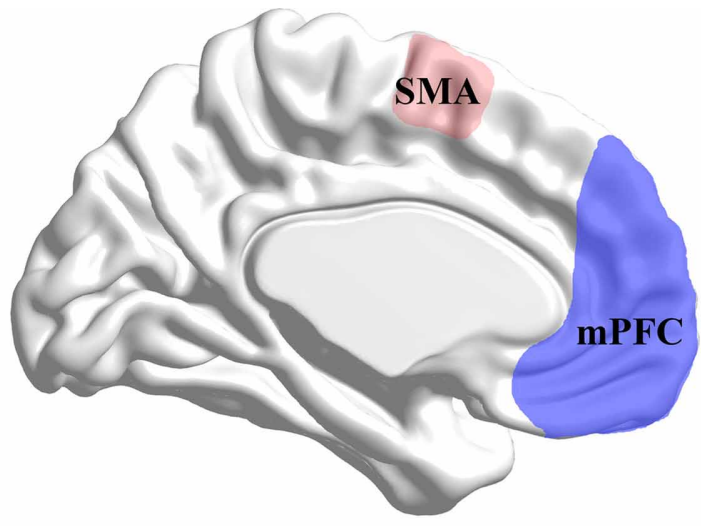

Extended AON Mentalizing System
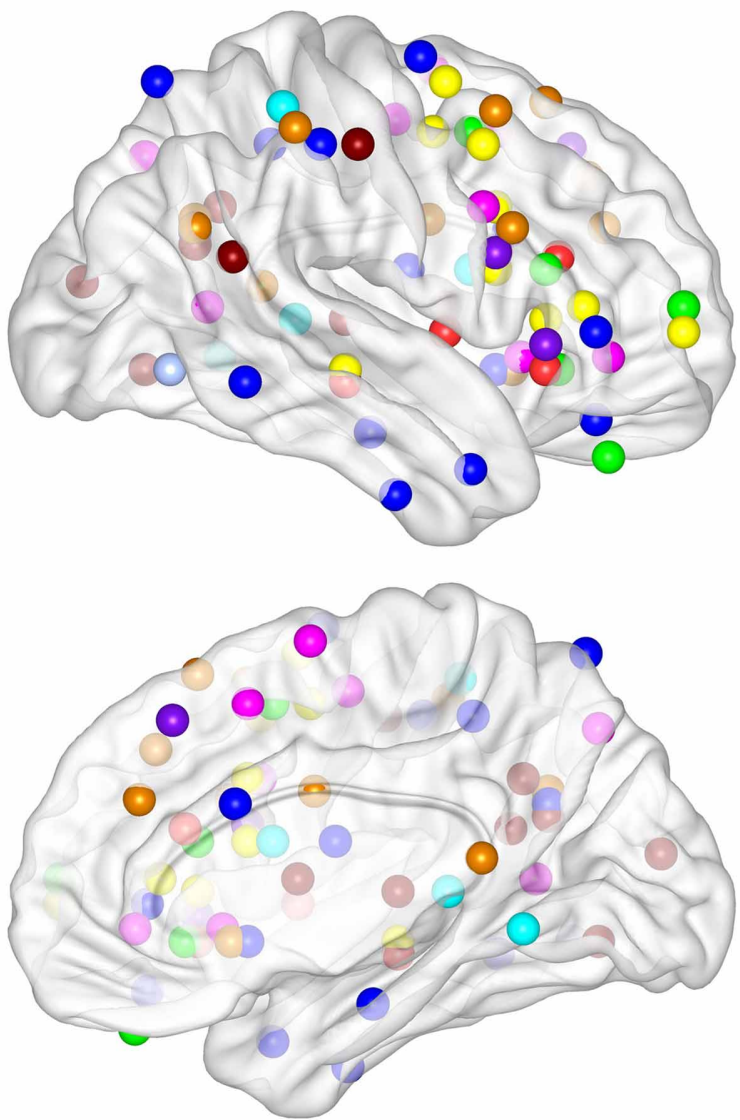

- Physical practice - Cross et al., 2006

- Physical practice - Cross et al., 2009a

Physical practice - Cross et al., 2009b

- Visual practice - Cross et al., 2009b

FIGURE 1 | Continued 
FIGURE 1 | (A) Schematic representation of the core AON, extended AON and mentalizing system. Three-dimensional representation of lateral and medial brain surface. The regions assigned to the "core" AON, "extended" AON and the "mentalizing" system are depicted. In red, the core AON is presented comprising: the PMV/pIFG complex, the aIPL and the STS. In pale red, the "extended" AON is presented comprising: the anterior part of the inferior frontal gyrus, (alFG), the dorsal premotor cortex (PMD), the supplementary motor area (SMA), the superior parietal lobule (SPL), the anterior intraparietal sulcus (AIP), the somatosensory cortex (S1) and the occipito-temporal cortex (OTC), including also STS. The mentalizing system (blue) is assumed to consist of the medial prefrontal cortex (mPFC) and the temporo-parietal junction (TPJ). Note that the extension of these networks is not representative of their real dimension or functional significance.

(B) Expertise effects. Three-dimensional representation of lateral and medial brain surface with location of peaks for the comparisons of interest superimposed. For Kim et al. (2011), we considered the comparison between the two groups (Table 2). For Wright et al. (2010), we considered results from ROI analysis (Table 2). For Wright et al. (2011), we plotted results for normal video (Table 2). For Abreu et al. (2012), we used the peak of the significant cluster within the temporal lobe in the group comparison (page 1649 of the manuscript). For Calvo-Merino et al. (2005), we plotted the reported interaction (see Table 1). For Calvo-Merino et al. (2006), we plotted the results from Table S2. For Cross et al. (2006), we considered the main effect of the contrast of interest (Table 2). For Cross et al. (2009a), we reported the contrast for physical training (Danced > Untrained) (Table 1). For Cross et al. (2009b), we considered the physical training results (Table 1) and the observational training results (Table 1). We excluded the peaks located within the cerebellum. dance moves, affects AON activity while observing the same movement.

Most of the contributions investigating motor expertise while observing sport actions are limited to one or few studies within the same domain, such as archery (Kim et al., 2011), badminton (Wright et al., 2010, 2011) or basketball (Abreu et al., 2012). Typically, these studies compare the blood-oxygen-level-dependent (BOLD) response between experts and novices. Although these studies considered different tasks and comparisons of experimental conditions, they seem to suggest a stronger activation for experts in comparison to novices not limited to the AON but recruiting also other brain regions (see Figure 1B). However, an interpretation of these results is difficult as, in addition to extensive practice of the observed movements, experts also have a strong visual familiarity with the observed stimuli which might affect the BOLD effect within the very same regions.

Beside these sparse investigations on different sport actions, a more systematic investigation involved the effect of dance expertise on activity within the AON (Calvo-Merino et al., 2005, 2006; Cross et al., 2006, 2009a,b, 2012, 2013; Pilgramm et al., 2010). Calvo-Merino et al. (2005) measured the BOLD effect of ballet dancers, capoeira dancers, and non-dancers watching two different types of dance movements (ballet or capoeira moves). They found a stronger recruitment of several regions within the AON (bilateral PMD, bilateral SPL and AIP, left PMV and left STS) in ballet and capoeira dancers for the observation of the trained in comparison to the untrained dance style, whereas they found no difference between the two dance styles in the nondancers.

Calvo-Merino et al. argued that the activation for the trained in comparison to the untrained dance style was due to simulation of those actions that were within the motor repertoire of the dancer. Alternatively, as pointed out above, dancers' strong visual familiarity with the observed stimuli might affect the measured difference in BOLD effect.

In a follow up study, Calvo-Merino et al. (2006) investigated this issue by trying to disentangle the different contributions of visual familiarity and motor practice on the BOLD effect within the AON of expert dancers. They exploited the fact that some ballet movements are gender-specific while others are commonly performed by both male and female dancers. Calvo-Merino et al. (2006) found that activity within several regions of the AON (left PMD, bilateral AIP) was higher when observing actions within the observer's motor repertoire. However, visual familiarity might have played a role also in this study since dancers might have gathered more visual experience with those movements that are part of their own motor repertoire.

Another series of studies by Cross et al. (2006, 2009a,b) explored how activity related to action observation is modified after the acquisition of motor (physical practice) and/or visual experience (visual practice) with specific dance actions. These authors demonstrated stronger activity within AON regions during the observation and imagination of observed actions which were previously trained physically in comparison to actions that were not (Cross et al., 2006). In a subsequent study
Cross et al. (2009a,b) showed that both previous physical and visual practice of dance sequences modulates activity within the AON while observing dance movements.

Figure 1B shows the peaks of activations for the different motor expertise studies. It is evident that there seems to be a consistent recruitment of premotor and parietal nodes of the AON for observing trained with respect to untrained moves, but, at the same time, there is also a widespread recruitment of other brain regions.

These studies suggest an effect of motor expertise on AON activation while perceiving an action, but it is difficult to assess the involvement of the AON in action understanding as none of these studies adopted a task directly investigating this process in a quantitative manner. Action understanding is intended here as the distinction between different actions irrespective of the properties (e.g., kinematics, goal, environmental cues, etc.) adopted to achieve such discrimination. We will elaborate on this point in the final section.

\section{FUTURE PERSPECTIVE: USING MOTOR EXPERTISE TO STUDY ACTION UNDERSTANDING}

In this section, we discuss possible ways of testing the proposed role of the AON in action understanding. If the ability to understand actions depends on sensorymotor representations of these actions, then an experience-based modification (either impairment or improvement) of these representations should lead to a corresponding measurable modification in the ability to understand these actions, as in tasks involving action recognition. 
Crucially, it is also necessary to discount the possible role of regions outside this network (e.g., the mentalizing system).

Motor expertise might serve as an interesting tool to test the involvement of areas within and outside the AON in action understanding. However, one of the problems to overcome is making sure that the learned movements were not previously experienced by the participants. As most everyday actions are physically or visually experienced during normal development, the new acquisition of complex movements, such as sport and dance moves, allows to more easily control for possible confounds related to previous exposure or practice of the studied movements. Another problem to face is that performance might be close to ceiling in tasks using natural stimuli (videos or pictures of actions), making it difficult to find a modulation of performance as a function of motor experience. One possibility to overcome this issue could be to use point-light display (Johansson, 1973). This stimulation recruits part of the AON (Saygin et al., 2004; Wright et al., 2011), and its perception has been shown to be affected by motor expertise (Casile and Giese, 2006). Furthermore, the adoption of point-light display might mitigate visual familiarity confounds as they do not resemble a "natural" stimulation, and they can be easily manipulated in order to disrupt the perceived movement simply by adding noise. A recent study (Lingnau and Petris, 2013) adopted this approach and observed that the ability to understand actions decreased with increasing noise level.

This approach could be adopted to investigate differences in action understanding, using point-light display with different level of noise, within the same individual on trained and untrained stimuli after different types of practice (as in Cross et al., 2006, 2009a,b). In addition to the possible effects of visual and physical practice, a motor-only training could be introduced where physical practice might be performed blindfolded in order to eliminate potential visual confounds (as in Casile and Giese, 2006). These different types of training might affect common or different parts of the brain during action understanding. Crucially, motor learning without visual feedback alone could determine a modification in action understanding performance and a related functional modification within or outside regions of the AON. This could demonstrate that motor learning alone might have an effect on visual recognition of trained actions, avoiding interpretational confounds induced by a concomitant visual learning.

We have highlighted motor expertise as an interesting experimental manipulation to comprehend the role of the AON in action understanding. Further, these studies will profit strongly from the adoption of new MVPA decoding techniques (Kriegeskorte and Bandettini, 2007) as they allow a more fine-grained distinction (e.g., between different types of observed or executed actions, see also Oosterhof et al., 2013) that are not possible to reveal with univariate methods. This could be especially useful to assess decoding accuracy modifications between different actions (e.g., move A vs. move B) based on the type of training (trained vs. untrained) with different levels of noise. Further, changes in decoding accuracy between different actions before and after training might be also informative regarding the regions affected by the different types of training (physical, visual or motor-only).

To conclude, this Opinion focused on describing neuroimaging investigations on action perception/understanding, which are correlational in nature. It is not possible to define a causal link between such results and concomitant behavioral changes. However, these studies might provide interesting starting points for future studies using TMS in healthy participants or voxel-based lesion-symptom mapping in brain damaged patients.

\section{ACKNOWLEDGMENTS}

This work was supported by a CARITRO grant of the Fondazione Cassa di Risparmio e Rovereto to Angelika Lingnau, and by the Provincia Autonoma di Trento.

\section{REFERENCES}

Abreu, A. M., Macaluso, E., Azevedo, R. T., Cesari, P., Urgesi, C., and Aglioti, S. M. (2012). Action anticipation beyond the action observation network: a functional magnetic resonance imaging study in expert basketball players. Eur. J. Neurosci. 35, 1646-1654. doi: 10.1111/j.14609568.2012.08104.x
Amodio, D. M., and Frith, C. D. (2006). Meeting of minds: the medial frontal cortex and social cognition. Nat. Rev. Neurosci. 7, 268-277. doi: 10.1038/nrn1884

Brass, M., Schmitt, R. M., Spengler, S., and Gergely, G. (2007). Investigating action understanding: inferential processes versus action simulation. Curr. Biol. 17, 2117-2121. doi: 10.1016/j.cub.2007.11.057

Calvo-Merino, B., Glaser, D. E., Grèzes, J., Passingham, R. E., and Haggard, P. (2005). Action observation and acquired motor skills: an FMRI study with expert dancers. Cereb. Cortex 15, 1243-1249. doi: 10.1093/cercor/bhi007

Calvo-Merino, B., Grèzes, J., Glaser, D. E., Passingham, R. E., and Haggard, P. (2006). Seeing or doing. influence of visual and motor familiarity in action observation. Curr. Biol. 16, 2277. doi: 10.1016/j.cub.2006.10.065

Casile, A., and Giese, M. A. (2006). Nonvisual motor training influences biological motion perception. Curr. Biol. 16, 69-74. doi: 10.1016/j.cub.2005.10.071

Caspers, S., Zilles, K., Laird, A. R., and Eickhoff, S. B. (2010). ALE meta-analysis of action observation and imitation in the human brain. Neuroimage 50, 1148-1167. doi: 10.1016/j.neuroimage.2009. 12.112

Chong, T. T.-J., Cunnington, R., Williams, M. A., Kanwisher, N., and Mattingley, J. B. (2008). fMRI adaptation reveals mirror neurons in human inferior parietal cortex. Curr. Biol. 18, 1576-1580. doi: 10.1016/j.cub.2008.08.068

Cross, E. S., Hamilton, A. F. D. C., and Grafton, S. T. (2006). Building a motor simulation de novo: observation of dance by dancers. Neuroimage 31, 1257-1267. doi: 10.1016/j.neuroimage.2006. 01.033

Cross, E. S., Hamilton, A. F. D. C., Kraemer, D. J. M., Kelley, W. M., and Grafton, S. T. (2009a). Dissociable substrates for body motion and physical experience in the human action observation network. Eur. J. Neurosci. 30, 1383-1392. doi: 10.1111/j.1460-9568.2009.06941.x

Cross, E. S., Kraemer, D. J. M., Hamilton, A. F. D. C., Kelley, W. M., and Grafton, S. T. (2009b). Sensitivity of the action observation network to physical and observational learning. Cereb. Cortex 19, 315-326. doi: 10.1093/cercor/bhn083

Cross, E. S., Liepelt, R., de C Hamilton, A. F., Parkinson, J., Ramsey, R., Stadler, W., et al. (2012). Robotic movement preferentially engages the action observation network. Hum. Brain Mapp. 33, 2238-2254. doi: 10.1002/hbm.21361

Cross, E. S., Stadler, W., Parkinson, J., SchützBosbach, S., and Prinz, W. (2013). The influence of visual training on predicting complex action sequences. Hum. Brain Mapp. 34, 467-486. doi: 10.1002/hbm. 21450

De Lange, F. P., Spronk, M., Willems, R. M., Toni, I., and Bekkering, H. (2008). Complementary systems for understanding action intentions. Curr. Biol. 18, 454-457. doi: 10.1016/j.cub.2008.02.057

Di Pellegrino, G., Di Fadiga, L., Fogassi, L., Gallese, V., and Rizzolatti, G. (1992). Understanding motor events: a neurophysiological study. Exp. Brain Res. 91, 176-180. doi: 10.1007/BF00230027

Dinstein, I., Hasson, U., Rubin, N., and Heeger, D. J. (2007). Brain areas selective for both observed 
and executed movements. J. Neurophysiol. 98, 1415-1427. doi: 10.1152/jn.00238.2007

Dinstein, I., Gardner, J. L., Jazayeri, M., and Heeger, D. J. (2008a). Executed and observed movements have different distributed representations in human aIPS. J. Neurosci. 28, 11231-11239. doi: 10.1523/ JNEUROSCI.3585-08.2008

Dinstein, I., Thomas, C., Behrmann, M., and Heeger, D. (2008b). A mirror up to nature. Curr. Biol. 18, 13-18. doi: 10.1016/j.cub.2007.11.004

Fogassi, L., Ferrari, P. F., Gesierich, B., Rozzi, S., Chersi, F., and Rizzolatti, G. (2005). Parietal lobe: from action organization to intention understanding. Science 308, 662-667. doi: 10.1126/science.1106138

Gallese, V., Fadiga, L., Fogassi, L., and Rizzolatti, G. (1996). Action recognition in the premotor cortex. Brain 119, 593-609. doi: 10.1093/brain/ 119.2.593

Gallese, V., Fadiga, L., Fogassi, L., and Rizzolatti, G. (2002). "Action representation and the inferior parietal lobule," in Common Mechanisms in Perception and Action Attention and Performance, Vol. XIX, eds W. Prinz and B. Hommel (Oxford: Oxford University Press), 247-266.

Gazzola, V., and Keysers, C. (2009). The observation and execution of actions share motor and somatosensory voxels in all tested subjects: single-subject analyses of unsmoothed fMRI data. Cereb. Cortex 19, 1239-1255. doi: 10.1093/cercor/ bhn181

Gazzola, V., Rizzolatti, G., Wicker, B., and Keysers, C. (2007). The anthropomorphic brain: the mirror neuron system responds to human and robotic actions. Neuroimage 35, 1674-1684. doi: 10.1016/j.neuroimage.2007.02.003

Hickok, G. (2009). Eight problems for the mirror neuron theory of action understanding in monkeys and humans. J. Cogn. Neurosci. 21, 1229-1243. doi: 10.1162/jocn.2009.21189

Hickok, G. (2013). Do mirror neurons subserve action understanding. Neurosci. Lett. 540, 56-58. doi: 10.1016/j.neulet.2012.11.001

Jacob, P., and Jeannerod, M. (2005). The motor theory of social cognition: a critique. Trends Cogn. Sci. 9, 21-25. doi: 10.1016/j.tics.2004.11.003

Johansson, G. (1973). Visual perception of biological motion and a model for its analysis. Percept. Psychophys. 14, 201-211. doi: 10.3758/BF03212378

Kilner, J. M., Neal, A., Weiskopf, N., Friston, K. J., and Frith, C. D. (2009). Evidence of mirror neurons in human inferior frontal gyrus. J. Neurosci. 29, 10153-10159. doi: 10.1523/JNEUROSCI.266809.2009

Kim, Y.-T., Seo, J.-H., Song, H.-J., Yoo, D.-S., Lee, H. J., Lee, J., et al. (2011). Neural correlates related to action observation in expert archers. Behav. Brain Res. 223, 342-347. doi: 10.1016/j.bbr.2011. 04.053

Kriegeskorte, N., and Bandettini, P. (2007). Analyzing for information, not activation, to exploit high-resolution fMRI. Neuroimage 38, 649-662. doi: 10.1016/j.neuroimage.2007.02.022
Lingnau, A., Gesierich, B., and Caramazza, A. (2009). Asymmetric fMRI adaptation reveals no evidence for mirror neurons in humans. Proc. Natl. Acad. Sci. U.S.A. 106, 9925-9930. doi: 10.1073/pnas.0902262106

Lingnau, A., and Petris, S. (2013). Action understanding within and outside the motor system: the role of task difficulty. Cereb. Cortex 23, 1342-1350. doi: 10.1093/cercor/bhs112

Mahon, B. Z., and Caramazza, A. (2008). A critical look at the embodied cognition hypothesis and a new proposal for grounding conceptual content. J. Physiol. 102, 59-70. doi: 10.1016/j.jphysparis.2008.03.004

Oosterhof, N. N., Tipper, S. P., and Downing, P. E. (2012). Viewpoint (in)dependence of action representations: an MVPA study. J. Cogn. Neurosci. 24, 975-989. doi: 10.1162/ jocn_a_00195

Oosterhof, N. N., Tipper, S. P., and Downing, P. E. (2013). Crossmodal and action-specific: neuroimaging the human mirror neuron system. Trends Cogn. Sci. (Regul. Ed.) 17, 311-318. doi: 10.1016/j.tics.2013.04.012

Oosterhof, N. N., Wiggett, A. J., Diedrichsen, J., Tipper, S. P., and Downing, P. E. (2010). Surface-based information mapping reveals crossmodal vision-action representations in human parietal and occipitotemporal cortex. J. Neurophysiol. 104, 1077-1089. doi: 10.1152/jn. 00326.2010

Van Overwalle, F. (2009). Social cognition and the brain: a meta-analysis. Hum. Brain Mapp. 30, 829-858. doi: 10.1002/hbm.20547

Van Overwalle, F., and Baetens, K. (2009). Understanding others' actions and goals by mirror and mentalizing systems: a metaanalysis. Neuroimage 48, 564-584. doi: 10.1016/j. neuroimage.2009.06.009

Pilgramm, S., Lorey, B., Stark, R., Munzert, J., Vaitl, D., and Zentgraf, K. (2010). Differential activation of the lateral premotor cortex during action observation. BMC Neurosci. 11:89. doi: 10.1186/14712202-11-89

Rizzolatti, G., Fogassi, L., and Gallese, V. (2001). Neurophysiological mechanisms underlying the understanding and imitation of action. Nat. Rev. Neurosci. 2, 1-10. doi: 10.1038/35090060

Rizzolatti, G., and Craighero, L. (2004). The mirrorneuron system. Annu. Rev. Neurosci. 27, 169-192. doi: 10.1146/annurev.neuro.27.070203.144230

Rizzolatti, G., and Sinigaglia, C. (2010). The functional role of the parieto-frontal mirror circuit: interpretations and misinterpretations. Nat. Rev. Neurosci. 11, 264-274. doi: 10.1038/nrn 2805

Saygin, A. P., Wilson, S. M., Hagler, D. J., Bates, E., and Sereno, M. I. (2004). Point-light biological motion perception activates human premotor cortex. J. Neurosci. 24, 6181-6188. doi: 10.1523/JNEUROSCI.0504-04.2004

Turella, L., Erb, M., Grodd, W., and Castiello, U. (2009a). Visual features of an observed agent do not modulate human brain activity during action observation. Neuroimage 46, 844-853. doi: 10.1016/j.neuroimage.2009. 03.002

Turella, L., Pierno, A. C., Tubaldi, F., and Castiello, U. (2009b). Mirror neurons in humans: consisting or confounding evidence. Brain Lang. 108, 10-21. doi: 10.1016/j.bandl.2007.11.002

Turella, L., Tubaldi, F., Erb, M., Grodd, W., and Castiello, U. (2012). Object presence modulates activity within the somatosensory component of the action observation network. Cereb. Cortex 22, 668-679. doi: 10.1093/cercor/ bhr140

Wright, M. J., Bishop, D. T., Jackson, R. C., and Abernethy, B. (2010). Functional MRI reveals expert-novice differences during sport-related anticipation. Neuroreport 21, 94-98. doi: 10.1097/WNR.0b013e328 333dff2

Wright, M. J., Bishop, D. T., Jackson, R. C., and Abernethy, B. (2011). Cortical fMRI activation to opponents' body kinematics in sportrelated anticipation: expert-novice differences with normal and point-light video. Neurosci. Lett. 500, 216-221. doi: 10.1016/j.neulet.2011. 06.045

Wurm, M. F., Cramon, D. Y., and Schubotz, R. I. (2012). The context-object-manipulation triad: cross talk during action perception revealed by fMRI. J. Cogn. Neurosci. 24, 1548-1559. doi: 10.1162/jocn_a_00232

Wurm, M. F., von Cramon, D. Y., and Schubotz, R. I. (2011). Do we mind other minds when we mind other minds' actions. A functional magnetic resonance imaging study. Hum. Brain Mapp. 32, 2141-2150. doi: 10.1002/hbm.21176

Wurm, M. F., and Schubotz, R. I. (2012). Squeezing lemons in the bathroom: contextual information modulates action recognition. Neuroimage 59, 1551-1559. doi: 10.1016/j.neuroimage.2011. 08.038

Received: 30 July 2013; accepted: 13 September 2013; published online: 16 October 2013.

Citation: Turella L, Wurm MF, Tucciarelli $R$ and Lingnau A (2013) Expertise in action observation: recent neuroimaging findings and future perspectives. Front. Hum. Neurosci. 7:637. doi: 10.3389/fnhum. 2013.00637

This article was submitted to the journal Frontiers in Human Neuroscience.

Copyright (־) 2013 Turella, Wurm, Tucciarelli and Lingnau. This is an open-access article distributed under the terms of the Creative Commons Attribution License (CC BY). The use, distribution or reproduction in other forums is permitted, provided the original author(s) or licensor are credited and that the original publication in this journal is cited, in accordance with accepted academic practice. No use, distribution or reproduction is permitted which does not comply with these terms. 